\title{
Nomograms incorporating preoperative RDW level for the prediction of postoperative complications and survival in colorectal liver metastases after resection
}

\author{
Qichen Chen, Rui Mao, Jianjun Zhao, Xinyu Bi, Zhiyu Li, Zhen Huang, Yefan Zhang, Jianguo Zhou, \\ Hong Zhao, Jianqiang Cai \\ Department of Hepatobiliary Surgery, National Cancer Center/National Clinical Research Center for Cancer/Cancer Hospital, Chinese Academy of \\ Medical Sciences and Peking Union Medical College, Beijing, China \\ Contributions: (I) Conception and design: H Zhao, J Cai; (II) Administrative support: H Zhao, J Cai; (III) Provision of study materials or patients: Q \\ Chen, R Mao; (IV) Collection and assembly of data: All authors; (V) Data analysis and interpretation: Q Chen; (VI) Manuscript writing: All authors; \\ (VII) Final approval of manuscript: All authors. \\ Correspondence to: Hong Zhao; Jianqiang Cai. Department of Hepatobiliary Surgery, National Cancer Center/National Clinical Research Center for \\ Cancer/Cancer Hospital, Chinese Academy of Medical Sciences and Peking Union Medical College, Beijing, China. \\ Email: pumczhaohong@126.com; caijianqiang188@sina.com.
}

Background: This study aimed to investigate the predictive significance of preoperative red cell volume distribution width (RDW) level for prognosis and to establish nomograms incorporating preoperative blood markers to predict postoperative complications and survival in patients with colorectal liver metastases (CRLM).

Methods: This retrospective study included 380 enrolled CRLM patients who underwent hepatic resection. Predictors of postoperative complications were explored using binary logistic regression analysis. Covariates associated with overall survival (OS) and progression-free survival (PFS) were evaluated through univariate and multivariate Cox regression analyses. Only variables that reached statistical significance at $\mathrm{P}<0.1$ in the univariate analysis were allowed to enter the multivariate analyses. The independent predictors that retained in the final multivariate model were incorporated into nomograms.

Results: The optimal cut-off point of preoperative RDW-CV was $16 \%$, and elevated RDW-CV was significantly associated with better prognosis (mPFS: 5.0 vs. 8.9 months, $\mathrm{P}=0.007$; mOS: 59.0 vs. 42.0 months, $\mathrm{P}=0.041$ ). The optimal cut-off point of preoperative RDW-SD was $43.9 \mathrm{fl}$, and elevated RDW$\mathrm{SD}$ was significantly associated with worse prognosis (mPFS: 8.0 vs. 13.0 months, $\mathrm{P}<0.001$; mOS:36.8 vs. 70.2 months, $\mathrm{P}=0.001)$. A nomogram predicting postoperative complications was constructed based on preoperative gamma-glutamyl transpeptidase (GGT) $\geq 34.5 \mathrm{U} / \mathrm{L}$, preoperative $\mathrm{RDW}-\mathrm{CV} \geq 14.1 \%$, and intraoperative blood loss $\geq 200.0 \mathrm{~mL}$, with AUROC of 0.658 . The calibration curves and HosmerLemeshow test revealed desirable model calibration (chi-square: 3.99, $\mathrm{P}=0.91$ ). A nomogram predicting PFS was constructed based on preoperative GGT $\geq 31.0 \mathrm{U} / \mathrm{L}$, preoperative D-dimer $\geq 0.251 \mathrm{mg} / \mathrm{L}$, preoperative $\mathrm{RDW}-\mathrm{CV}<16.0 \%$, preoperative $\mathrm{RDW}-\mathrm{SD} \geq 43.9 \mathrm{fl}$, positive lymph node metastasis, bilobar liver distribution, and R0 resection with good discrimination (C-index: 0.676 0.016$)$ and calibration. A nomogram for the prediction of OS was constructed with favorable discrimination (C-index: $0.700 \pm 0.021$ ) and calibration. Significant differences in PFS and OS were shown among patients stratified into three different risk groups $(\mathrm{P}<0.001)$ based on the nomograms.

Conclusions: This study first revealed the relationship between preoperative RDW-SD, RDW-CV, and prognosis in patients with CRLM. It also established nomograms especially considering preoperative blood markers to predict postoperative complications, PFS, and OS, which facilitated physicians to determine the optimal clinical management strategies.

Keywords: Colorectal liver metastases; red cell volume distribution width (RDW); nomogram; prognosis 
Submitted Dec 03, 2020. Accepted for publication Feb 03, 2021.

doi: 10.21037/apm-20-2418

View this article at: http://dx.doi.org/10.21037/apm-20-2418

\section{Introduction}

Colorectal cancer (CRC) is the third most common malignancy with more than 1.8 million new cases worldwide per year, and it accounts for $9 \%$ of all cancerrelated deaths (1). Liver is the predominant site of distant metastases with $25-30 \%$ of CRC patients developing metastases in this organ $(2,3)$. Liver resection of colorectal liver metastasis (CRLM) offers the best chance for a cure and long-term survival, as it has a five-year survival of up to $47-60 \%$. Recurrences occur in $40-75 \%$ of patients with CRLM $(2,4)$ and the incidence of postoperative complications is approximately $38-54 \%(5,6)$. Therefore, effective prediction of the survival of patients with CRLM in this specific clinical scenario is urgently needed. Accurate prognostic assessment helps manage appropriate treatment and follow-up plans. Nomograms incorporating and illustrating important prognostic factors have been widely used as reliable tools for predicting survival probabilities for individual patients. Chen et al. (7) established a comprehensive evaluation of relapse risk score based on KRAS status, primary node status, extrahepatic disease, carcinoembryonic antigen (CEA) level, and tumour burden score to predict the prognosis of patients with CRLM. Liu et al. (8) revealed that a nomogram constructed according to tumour size, liver metastasis number, RAS mutation status, and primary lymph node metastasis has a favorable calibration and $\mathrm{C}$-index for predicting survival in patients with CRLM.

In recent years, various blood markers with different changes were hypothesized to have a relationship with prognosis of malignant tumour diseases. Previous findings demonstrated that high serum gamma-glutamyl transpeptidase (GGT) levels and D-dimer levels were significantly associated with worse prognosis in lung cancer, hepatocellular carcinoma, etc. (9-12). It has been reported that the red cell volume distribution width (RDW) level of patients with malignant tumours including rectal cancer, colon cancer and bladder cancer is correlated with diagnosis, staging, and prognosis (13-15). However, no study has revealed the relationship between RDW level and prognosis in patients with CRLM after liver resection, and no nomogram has incorporated preoperative blood markers. Therefore, the aim of this study was as follows:
(I) to investigate the predictive significance of preoperative RDW level for prognosis; (II) to establish nomograms incorporating preoperative blood markers to predict postoperative complications and survival in patients with CRLM. We hypothesized that nomograms incorporating preoperative RDW level will enhance the predictive ability and facilitate physicians to determine the optimal clinical management strategies for CRLM patients. We present the following article in accordance with the STROBE reporting checklist (available at http://dx.doi.org/10.21037/apm-202418).

\section{Methods}

\section{Patients}

There were 380 enrolled CRLM patients who underwent hepatic resection between February 2009 and February 2018 in Cancer Hospital, Chinese Academy of Medical Sciences. This study was conducted with approval from the Institute Research Ethics Committee of the Cancer Hospital, Chinese Academy of Medical Sciences (ID: NCC2019C-016). The study conformed to the provisions of the Declaration of Helsinki (as revised in 2013). Informed consents from patients have been obtained. Eligibility criteria were: (I) histologically proven colorectal adenocarcinoma liver metastases; (II) patients underwent hepatic resection and colorectal resection for therapeutic purpose. The exclusion criteria were: (I) with other malignancies; (II) loss to follow-up or incomplete clinical data. Flow diagram for the selection of CRLM included in the final analyses of this study was shown in Figure 1. By using peripheral venous punctures, blood samples for the evaluation of serum testing markers were obtained. Preoperative serum GGT levels (normal range: 0-55.0 U/L), D-dimer levels (normal range: $0-0.550 \mathrm{mg} / \mathrm{L}$ ), RDW-CV (normal range: 11.6-14.6\%) and RDW-SD (normal range: $37.0-51.0 \mathrm{fl}$ ) were measured within 1 week before surgery.

\section{Treatment}

It was recommended to receive neoadjuvant chemotherapy (NAC) for CRLM patients if there were some highrisk factors for recurrence (16). The NAC regimens 


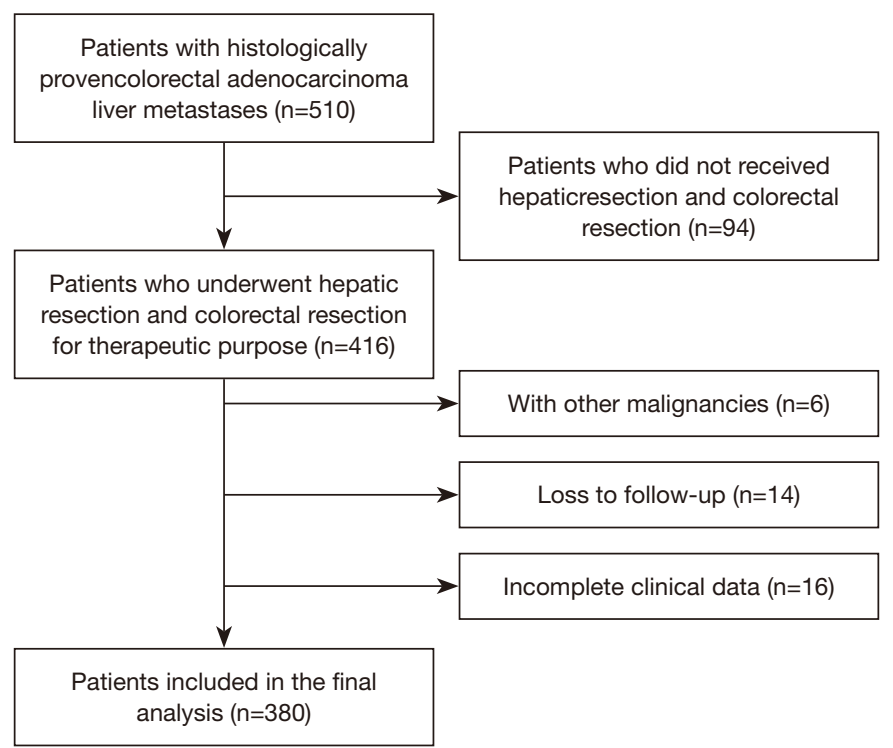

Figure 1 Flow diagram for the selection of CRLM patients. CRLM, colorectal liver metastases.

recommended include FOLFOX (5-fluorouracil+oxaliplatin), FOLFIRI (5-fluorouracil+irinotecan), and CapeOX (capecitabine+oxaliplatin). The targeted therapy regimens included bevacizumb and cetuximab. Patients usually underwent liver resections within 4-6 weeks after the completion of NAC. Liver resections were defined as major liver resection (resections of more than two segments) or minor liver resections. According to the Clavien system (17), postoperative complications were graded, and major complications were defined as grade III or IV complications. The highest grade was used when patients experienced multiple postoperative complications. The postoperative complications were also defined as surgery-related complications (gastrointestinal tract leak, gastrointestinal tract necrosis, intrathoracic or intraabdominal abscess, hemorrhage, ileus, et al.) and general complications (pulmonary, cardiac, hepatic, renal complications, and catheter sepsis) (18). The clinical risk-scoring (CRS) scores were defined as follows: number of CRLM more than 1 (1 point); maximum CRLM diameter more than $5 \mathrm{~cm}$ (1 point); CEA level $>200.0 \mathrm{ng} / \mathrm{mL}$ (1 point); primary lymph node positive (1 point); a disease-free interval of less than 12 months after the diagnosis of primary CRC (1 point).

\section{Follow-up and outcomes}

Patients were followed up at regular intervals after surgery.
The first follow-up date was one month after surgery, with subsequent ones every 3 months in 2 years, every 6 months between 2-5 years, and every one year thereafter. Overall survival (OS) was defined as the interval from the date of resection to death or the last follow-up. Progression-free survival (PFS) was defined as the interval from the date of resection to progression or the last follow-up.

\section{Statistical analysis}

To analyze continuous variables, the Mann-Whitney $\mathrm{U}$ test was implemented. And categorical variables were analyzed by the Chi-square or Fisher's exact test. X-tile analysis was implemented to investigate the optimal cut-off values of the preoperative testing markers for survival (19). The optimal cutoff values for post-operative complications were identified by the highest Youden index (sensitivity+specificity-1). To determine the optimal segmentation threshold for survival curves risk stratification, the $\mathrm{X}$-tile analysis was conducted.

The OS and PFS were measured by the Kaplan-Meier method and compared with the log-rank test Factors predictive of postoperative complications were explored using binary logistic regression analysis. Covariates associated with OS and PFS were evaluated through univariable and multivariate Cox regression analyses. Briefly, as the sample included in this study is limited, only variables that reached statistical significance at $\mathrm{P}<0.1$ in the univariate 
analysis were allowed to enter the multivariate analyses. Forward method was used to select the final predictors. The independent predictors that retained in the final model were incorporated into a nomogram. The C-index and area under the receiver operating characteristic curve (AUROC) was used to evaluate the discrimination of the nomogram. The calibration plots and the Hosmer-Lemeshow chisquare test were performed to assess the calibration. The decision curve analysis (DCA) was conducted to determine the clinical usefulness by quantifying the net benefits at different threshold probabilities. SPSS version 22 software (Armonk NV, USA) and R software (http://www.r-project. org) were used to perform statistical analysis. Statistical significance was defined as $\mathrm{P}<0.05$.

\section{Results}

\section{Clinicopatbological characteristics}

Three hundred and eighty patients were included in this study, including 144 females and 236 males with an average age of $56.8 \pm 10.0$ years. Primary tumours located in the colon were found in 205 patients (53.9\%). Bilobar distribution of liver metastases was found in 133 patients (35.0\%). The median diameter of the largest liver metastasis was $2.6 \mathrm{~cm}$ [interquartile range (IQR): 1.8-4.0]. The median number of liver metastases was 2.0 (IQR: 1.0-3.0). Extrahepatic metastases were observed in 46 patients $(12.1 \%)$. Primary lymph node metastasis of the primary tumour was observed in $69.5 \%$ of the patients. Synchronous metastasis was observed in 291 patients (76.6\%). A CRS score of 3-5 was observed in 158 patients. One hundred and fifty patients $(39.5 \%)$ underwent major liver resection. ASA class 1-2 was observed in 339 patients (89.2\%). The median operation time was $283.5 \mathrm{~min}$ (IQR: 180.0-370.0), and the median intraoperative blood loss was $200.0 \mathrm{~mL}$ (IQR: 100.0-400.0). A total of 197 patients $(51.8 \%)$ received NAC; 136 patients $(69.0 \%)$ received an oxaliplatinbased NAC regimen, 30 patients $(15.2 \%)$ received an irinotecan-based regiment and 31 patients $(15.8 \%)$ received oxaliplatin-based regimen combined with irinotecan-based regiment. Sixty-six patients (33.5\%) underwent targeted therapy (bevacizumab: 31 patients; cetuximab: 34 patients; bevacizumab + cetuximab: 1 patient). The median NAC cycles was 5.0 (IQR: 3.0-7.0). One hundred and ninetythree patients underwent postoperative chemotherapy. The preoperative median $\mathrm{RDW}-\mathrm{SD}$ value was $45.6 \mathrm{fl}$ (IQR: 41.0-51.8). The preoperative median $\mathrm{RDW}-\mathrm{CV}$ value was
13.7\% (IQR: 12.7-15.5). The preoperative median GGT value was $31.0 \mathrm{U} / \mathrm{L}$ (IQR: 20.0-50.0). The preoperative median D-dimer value was $0.370 \mathrm{mg} / \mathrm{L}$ (IQR: $0.220-0.630$ ). The demographic and clinical characteristics of the patients are listed in Table 1.

\section{Predictors for postoperative complications}

In this study, 179 patients $(47.11 \%)$ experienced postoperative complications (surgery-related complications: 58 patients, general complications: 87 patients, and surgeryrelated complications combined with general complications: 34 patients), including 68 major complications and 111 minor complications. According to the highest Youden index, the optimal cut-off points of preoperative RDW-CV level, RDW-SD level, D-dimer level, and GGT level were $14.1 \%, 48.6 \mathrm{fl}, 0.485 \mathrm{mg} / \mathrm{L}$, and $34.5 \mathrm{U} / \mathrm{L}$, respectively. The elevated RDW-CV level, RDW-SD level, D-dimer level, and GGT level were significantly associated with occurrence of postoperative complications.

In the univariate analysis, preoperative serum, preoperative GGT $\geq 34.5 \mathrm{U} / \mathrm{L}(\mathrm{P}=0.001)$, preoperative $\mathrm{D}$-dimer $\geq 0.485 \mathrm{mg} / \mathrm{L}(\mathrm{P}=0.032)$, preoperative $\mathrm{RDW}$ $\mathrm{CV} \geq 14.1 \%(\mathrm{P}<0.001)$, preoperative $\mathrm{RDW}-\mathrm{SD} \geq 48.6 \mathrm{fl}$ $(\mathrm{P}=0.004)$, NAC $(\mathrm{P}<0.001)$, diameter of liver metastases $\geq 3.0 \mathrm{~cm}(\mathrm{P}=0.005)$, major liver resection $(\mathrm{P}=0.005)$, operation time $\geq 283.5 \mathrm{~min}(\mathrm{P}=0.010)$, and intraoperative blood loss $\geq 200.0 \mathrm{~mL}(\mathrm{P}=0.001)$ were significantly associated with postoperative complications. The predictors $(\mathrm{P}<0.1)$ were included in the multivariate analysis, and preoperative GGT $\geq 34.5 \mathrm{U} / \mathrm{L}(\mathrm{OR}=1.811,95 \% \mathrm{CI}$ : 1.178-2.786, $\mathrm{P}=0.007)$, preoperative $\mathrm{RDW}-\mathrm{CV} \geq 14.1 \%$ $(\mathrm{OR}=1.590,95 \% \mathrm{CI}: 1.001-2.526, \mathrm{P}=0.049)$, and intraoperative blood loss $\geq 200.0 \mathrm{~mL}$ ( $\mathrm{OR}=1.739,95 \%$ CI: $1.113-2.716, \mathrm{P}=0.015)$ were independently associated with the presence of postoperative complications (Table 2).

\section{Nomogram for the prediction of postoperative complications}

A nomogram with three independent predictors from the multivariate analysis was developed (Figure 2). These factors were assigned specific scores as follows: preoperative serum GGT $\geq 34.5$ U/L, 100; preoperative RDW-CV $\geq 14.1 \%$, 94; and intraoperative blood loss $\geq 200.0 \mathrm{~mL}, 94$. The total risk score of each patient based on the nomogram was calculated, and it ranged from 0 to 288 . The cutoff value was set at 144 according to the ROC curve, with a sensitivity of 0.631 and a specificity of 0.632 . The 
Table 1 Characteristics of CRLM patients after liver resection

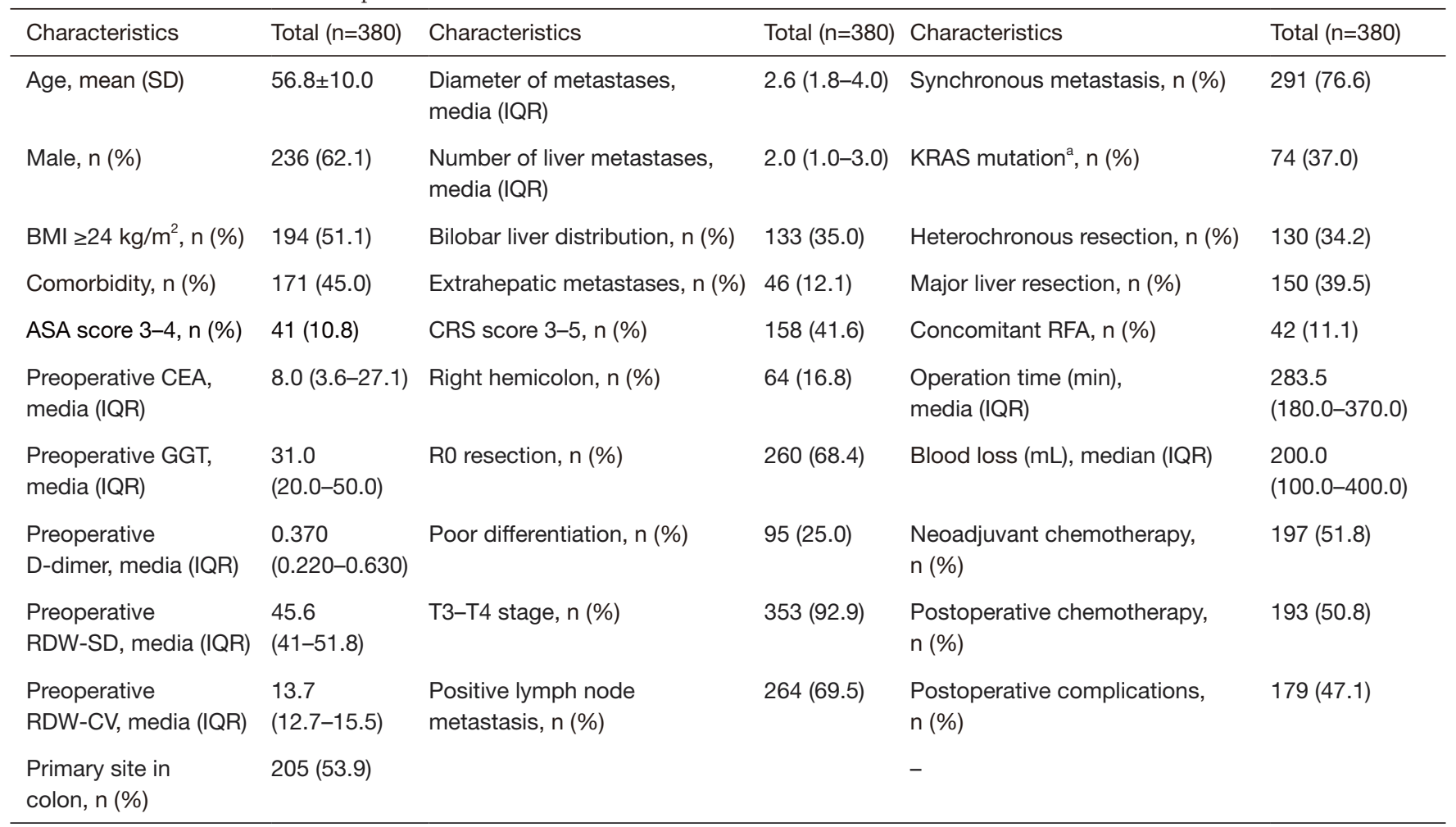

${ }^{a}$ KRAS status was available in 200 patients; CRLM, colorectal liver metastases; CEA, carcinoembryonic antigen; GGT, gamma-glutamy Itranspeptidase; RDW, red cell volume distribution width; IQR, interquartile range; CRS, clinical risk-scoring; RFA, radiofrequency ablation.

performance of the model was desirable with an AUROC of 0.658 (95\% CI: 0.604-0.712) (Figure 3A). Calibration curves and the Hosmer-Lemeshow test demonstrated acceptable model calibration (chi-square: 3.99, $\mathrm{P}=0.91$ ) (Figure 3B). DCA revealed that using this nomogram was probably more beneficial (Figure 3C).

\section{Best cut-off point of preoperative serum testing markers for survival}

The optimal cut-point of preoperative $\mathrm{RDW}-\mathrm{CV}$ was $16.0 \%$, and elevated RDW-CV was significantly associated with better prognosis (mPFS: 15.0 vs. 8.9 months, $\mathrm{P}=0.007$; mOS: 59.0 vs. 42.0 months, $\mathrm{P}=0.041$ ) (Figure $4 A, B$ ). The optimal cut-point of preoperative RDW-SD was $43.9 \mathrm{fl}$, and elevated RDW-SD was significantly associated with worse prognosis (mPFS: 8.0 vs. 13.0 months, $\mathrm{P}<0.001$; mOS: 36.8 vs. 70.2 months, $\mathrm{P}=0.001$ ) (Figure $4 C, D$ ). Optimal cut-point of preoperative GGT was $31.0 \mathrm{U} / \mathrm{L}$, and elevated GGT was significantly associated with worse prognosis (mPFS: $7.1 \mathrm{vs}$. 13.0 months, $\mathrm{P}<0.001$; mOS: 36.0 vs. 57.7 months, $\mathrm{P}=0.001$ ).
The optimal cut-point of preoperative Di-dimer was $0.251 \mathrm{mg} / \mathrm{L}$, and elevated Di-dimer was significantly associated with worse PFS (mPFS: 8.4 vs. 14.0 months, $\mathrm{P}=0.003$ ) and an equivalent OS (mPFS: 42.0 vs. 54.8 months, $\mathrm{P}=0.330)$.

\section{Analysis of CRS score for survival}

The Kaplan-Meier analysis of PFS and OS for different values of the CRS score is shown in Figure 5. Patients were stratified by their CRS score into the high-risk group (CRS score 3-5) and low-risk group (CRS score 0-2). Patients in the high-risk group exhibited worse PFS (mPFS: 7.0 vs. 12.0 months, $\mathrm{P}<0.001)$ and worse $\mathrm{OS}(\mathrm{mOS}: 35.2$ vs. 57.7 months, $\mathrm{P}<0.001$ ) (Figure 5).

\section{Prognostic factors for PFS}

In this study, 286 patients (75.26\%) experienced tumour progression. The median PFS was 10.0 months (IQR: 8.6-11.4), and the 1-, 3-, and 5-year PFS rates were 
Table 2 Prognostic factors for post-operative complications in CRLM patients after liver resection

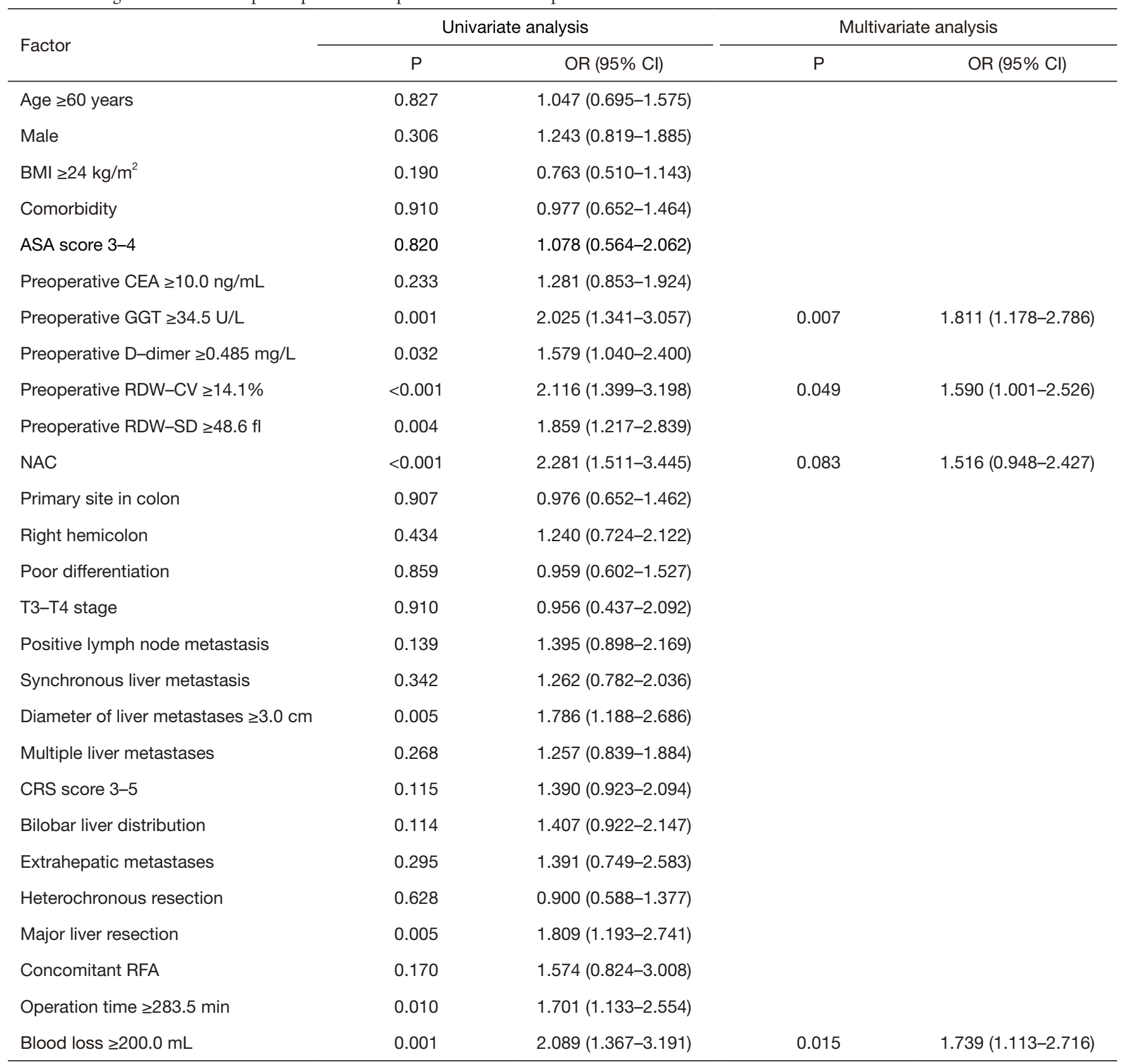

CRLM, colorectal liver metastases; CEA, carcinoembryonic antigen; GGT, gamma-glutamy Itranspeptidase; RDW, red cell volume distribution width; NAC, neoadjuvant chemotherapy; CRS, clinical risk-scoring; RFA, radiofrequency ablation.

$43.9 \%, 23.1 \%$, and $22.4 \%$, respectively. In the univariable analysis, preoperative CEA $\geq 10.0 \mathrm{ng} / \mathrm{mL}$, preoperative GGT $\geq 31.0 \mathrm{U} / \mathrm{L}$, preoperative D-dimer $\geq 0.251 \mathrm{mg} / \mathrm{L}$, preoperative $\mathrm{RDW}-\mathrm{CV}<16.0 \%$, preoperative $\mathrm{RDW}-\mathrm{SD}$ $\geq 43.9 \mathrm{fl}, \mathrm{T} 3-\mathrm{T} 4$ stage, positive lymph node metastasis, multiple liver metastases, bilobar liver distribution, extrahepatic metastases, synchronous liver metastasis, non-R0 resection, major liver resection, operation time $\geq 283.5 \mathrm{~min}$, complications, and NAC were related to decreased PFS $(\mathrm{P}<0.05)$.

Seven independent prognostic factors for PFS were identified in the multivariable analysis: preoperative GGT 


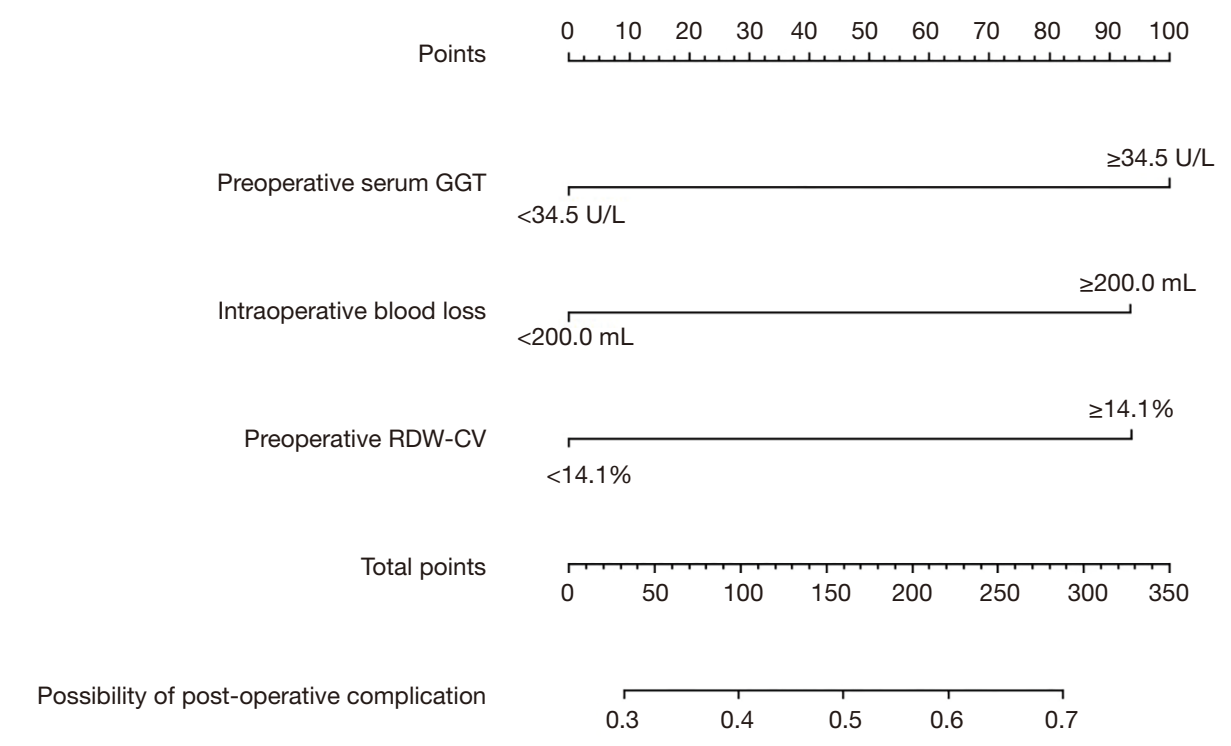

Figure 2 Nomogram predicting the probability of post-operative complications in CRLM patients. CRLM, colorectal liver metastases.
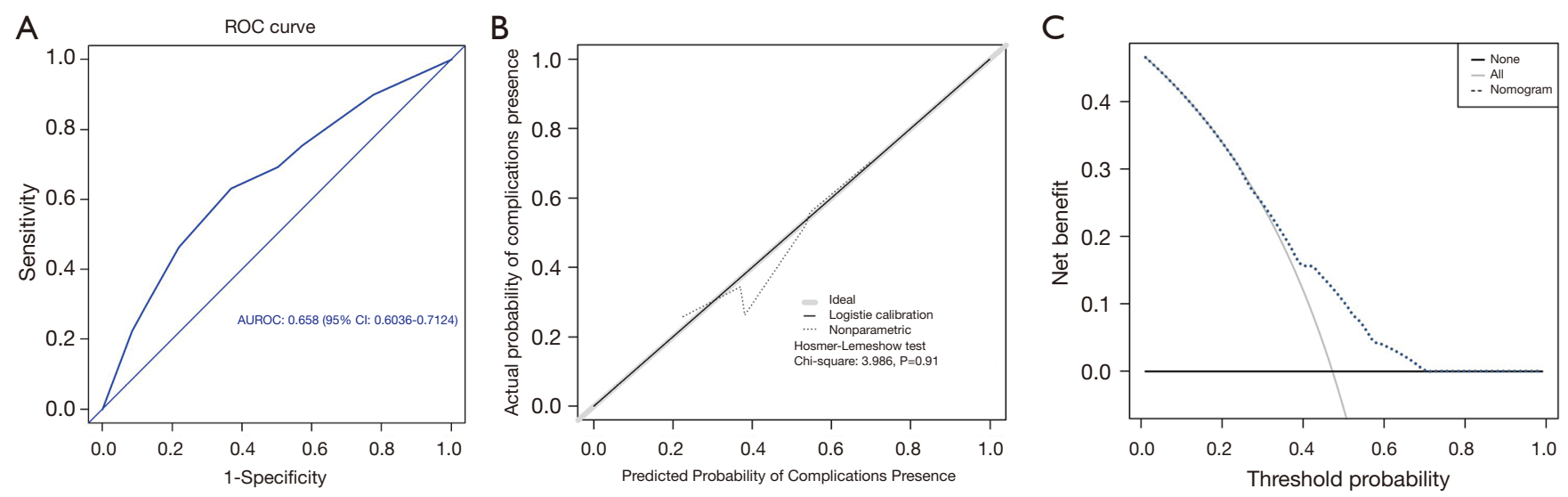

Figure 3 Evaluation of nomogram in the prediction of post-operative complications. (A) The ROC curves of the nomogram. (B) The calibration curves for predicting post-operative complications presence. (C) DCA for the nomogram. ROC, receiver operating characteristic curve; DCA, decision curve analysis.

$\geq 31.0 \mathrm{U} / \mathrm{L}$ (HR $=1.369,95 \%$ CI: 1.074-1.744, $\mathrm{P}=0.011$ ), preoperative D-dimer $\geq 0.251 \mathrm{mg} / \mathrm{L}$ (HR $=1.425,95 \%$ CI: 1.081-1.877, $\mathrm{P}=0.012)$, preoperative $\mathrm{RDW}-\mathrm{CV} \geq 16.0 \%$ (HR $=0.516,95 \%$ CI: $0.373-0.713, \mathrm{P}<0.001$ ), preoperative RDW-SD $\geq 43.9$ fl (HR $=1.533,95 \%$ CI: $1.170-2.009$, $\mathrm{P}=0.002)$, positive lymph node metastasis $(\mathrm{HR}=1.433$, 95\% CI: $1.082-1.899, \mathrm{P}=0.012$ ), bilobar liver distribution ( $\mathrm{HR}=1.391,95 \% \mathrm{CI}: 1.068-1.814, \mathrm{P}=0.015)$ and $\mathrm{R} 0$ resection (HR $=0.629,95 \%$ CI: $0.483-0.819, \mathrm{P}=0.001)$ (Table 3).

\section{Nomogram for PFS prediction}

A prognostic nomogram for PFS with point scales for the above seven independent prognostic factors was established (Figure 6). These factors were assigned specific scores as follows: preoperative GGT $\geq 31.0 \mathrm{U} / \mathrm{L}, 47$; preoperative D-dimer $\geq 0.251 \mathrm{mg} / \mathrm{L}, 53$; preoperative $\mathrm{RDW}-\mathrm{CV}<16.0 \%$, 100; preoperative $\mathrm{RDW}-\mathrm{SD} \geq 43.9 \mathrm{fl}, 64$; positive lymph node metastasis, 54; bilobar liver distribution, 49; non $\mathrm{R} 0$ resection, 70. The C-statistic for PFS prediction was 
A

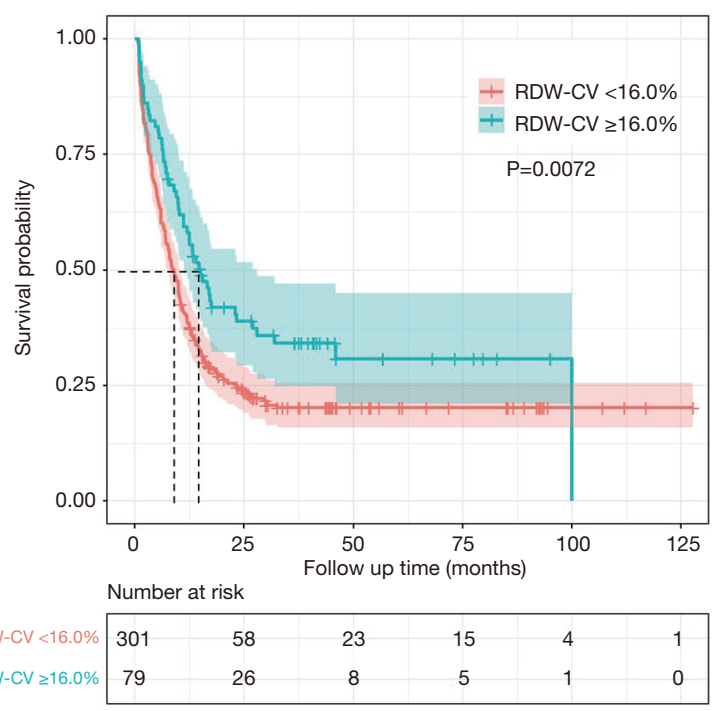

\section{C}

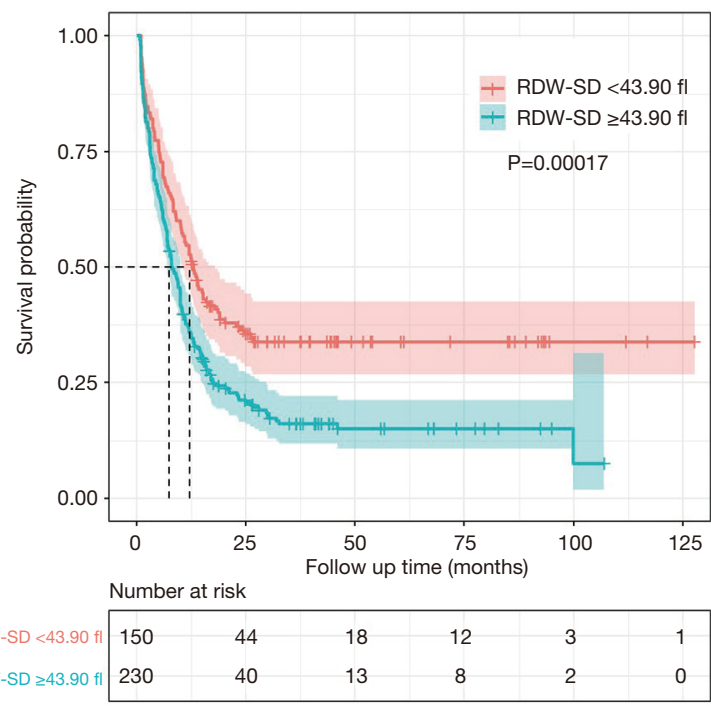

B
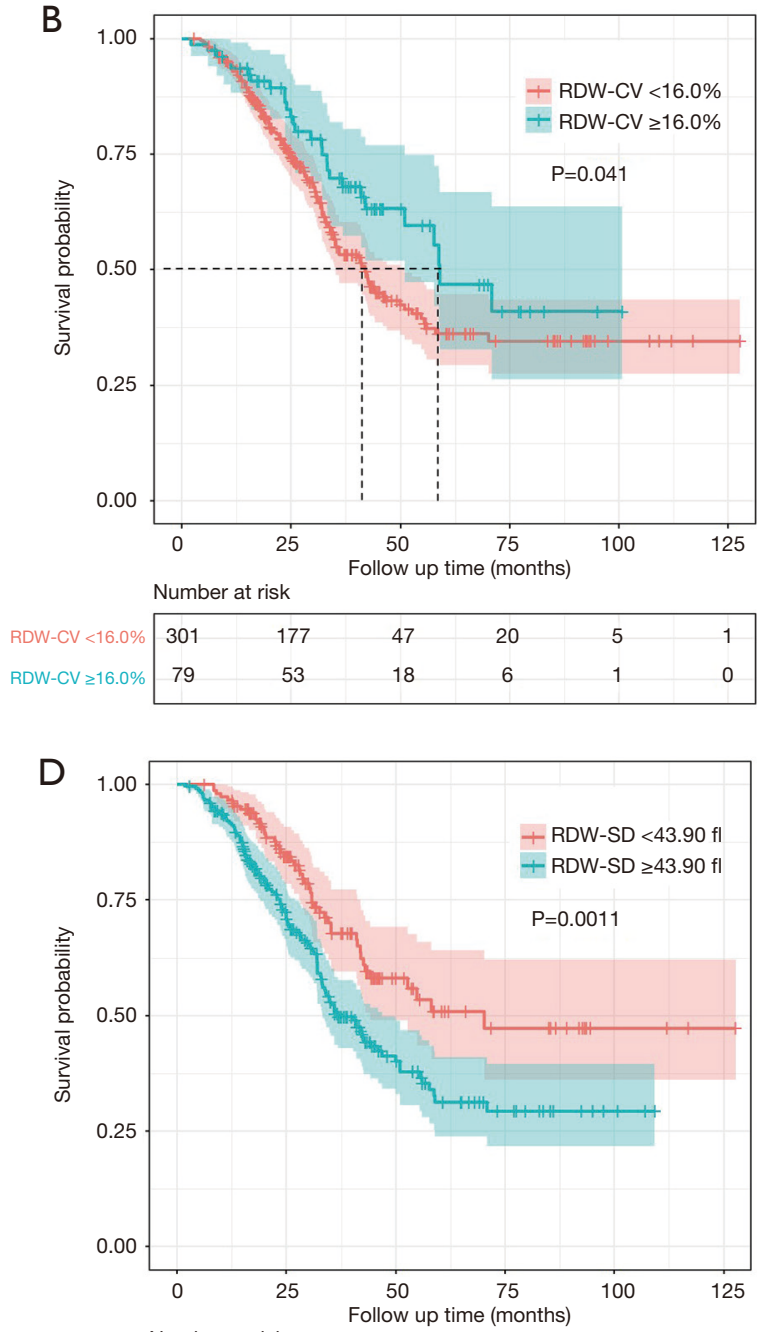

\begin{tabular}{|c|c|c|c|c|c|c|}
\hline \multirow[b]{2}{*}{ RDW-SD $<43.90 \mathrm{fl}$} & \multicolumn{6}{|c|}{ Number at risk } \\
\hline & 150 & 98 & 28 & 12 & 3 & 1 \\
\hline RDW-SD $\geq 43.90 \mathrm{fl}$ & 230 & 132 & 37 & 14 & 3 & 0 \\
\hline
\end{tabular}

Figure 4 Survival analysis of RDW level. (A) PFS analysis of RDW-CV $<16.0 \%$ versus RDW-CV $\geq 16.0 \%$. (B) $\mathrm{OS}$ of RDW-CV $<16.0 \%$ versus $\mathrm{RDW}-\mathrm{CV} \geq 16.0 \%$. (C) PFS analysis of RDW-SD $<43.9 \mathrm{fl}$ versus RDW-SD $\geq 43.9 \mathrm{fl}$. (D) $\mathrm{OS}$ analysis of RDW-SD $<43.9 \mathrm{fl}$ versus $\mathrm{RDW}-\mathrm{SD} \geq 43.9 \mathrm{fl}$. RDW, red cell volume distribution width; PFS, progression-free survival; OS, overall survival.

$0.676 \pm 0.016$, which was significantly greater than that of the CRS score $(0.676$ vs. $0.564, \mathrm{P}<0.001)$. A calibration plot for the probability of PFS at 1, 3 and 5 years (Figure 7) demonstrated good calibration between the predictions by the nomogram and the actual observations.

We further calculated the total risk scores of each patient based on the nomogram, and the total points for the scores ranged from 0 to 500. According to the total risk scores, $\mathrm{X}$-tile analysis was conducted to determine the optimal segmentation threshold for dividing patients into three subgroups \{high-risk [321-437], middle-risk [219-320] and low-risk [0-218] groups\}. As shown in Figure 5, the high-risk group was associated with significantly worse PFS than the middle-risk group $(\mathrm{P}<0.001 ;$ mPFS: 4.7 vs. 7.7 months) and the low-risk group $(\mathrm{P}<0.001$; mPFS: 4.7 vs. 20.2 months). The middle-risk group exhibited significantly worse PFS than the low-risk group $(\mathrm{P}<0.001$; mPFS: 7.7 vs. 20.2 months). 

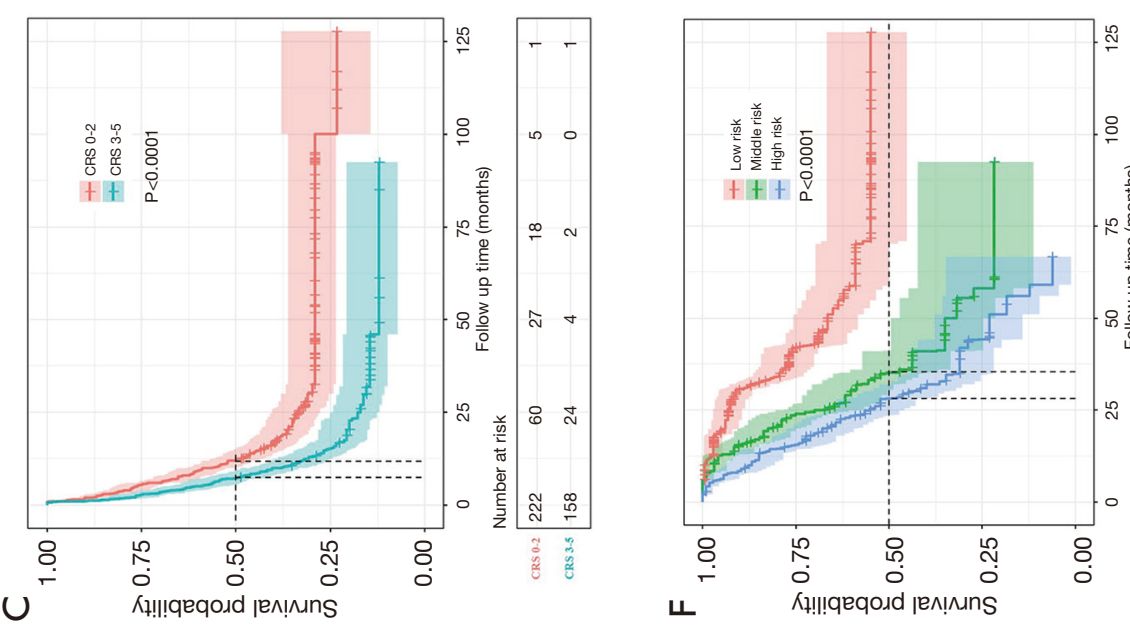

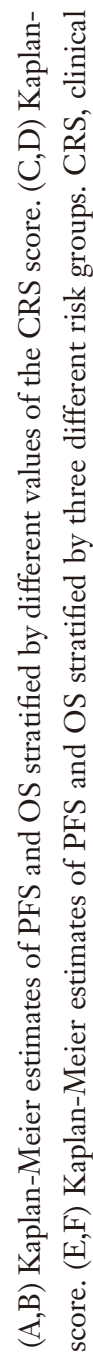
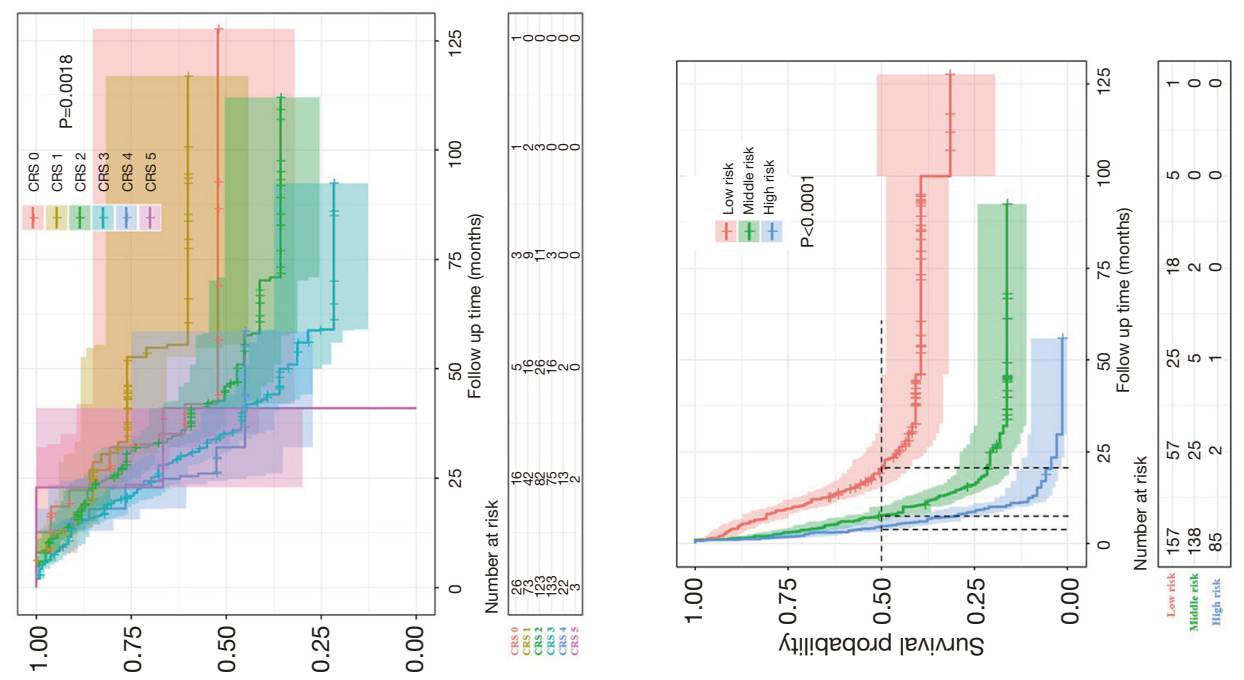

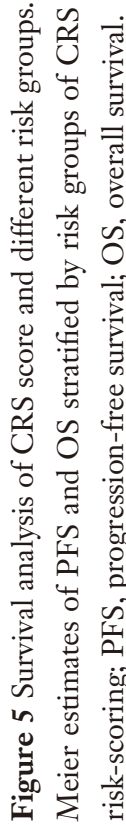

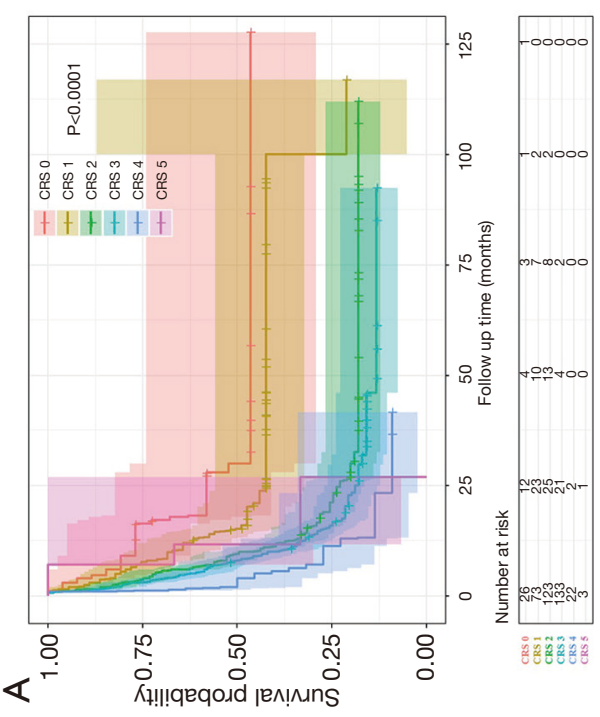

ш

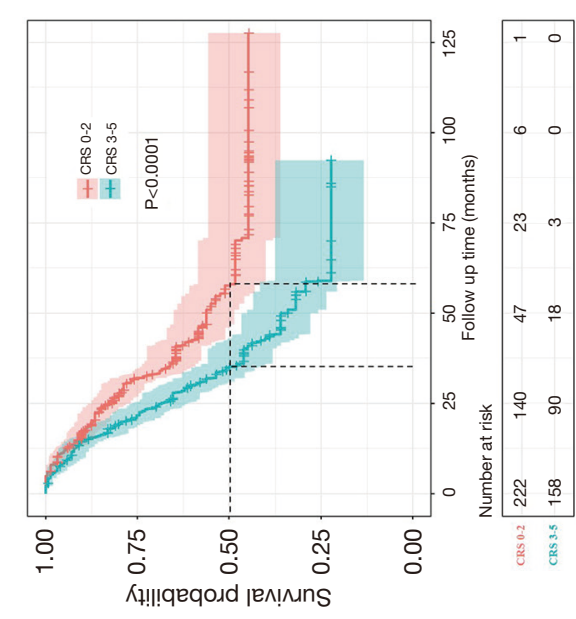

๑
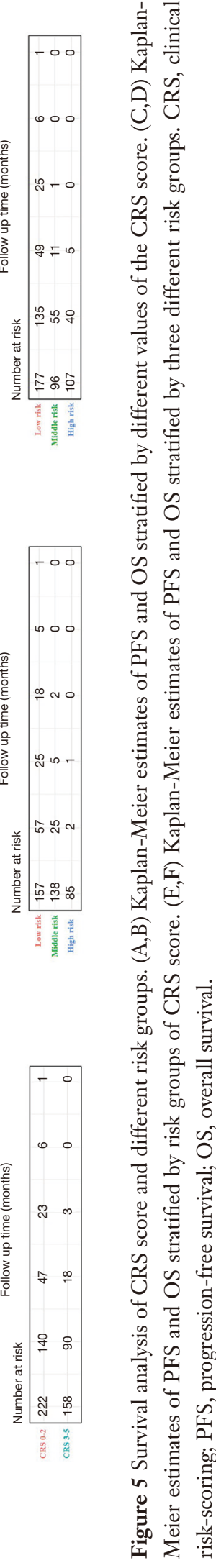
Table 3 Prognostic factors for PFS in CRLM patients after liver resection

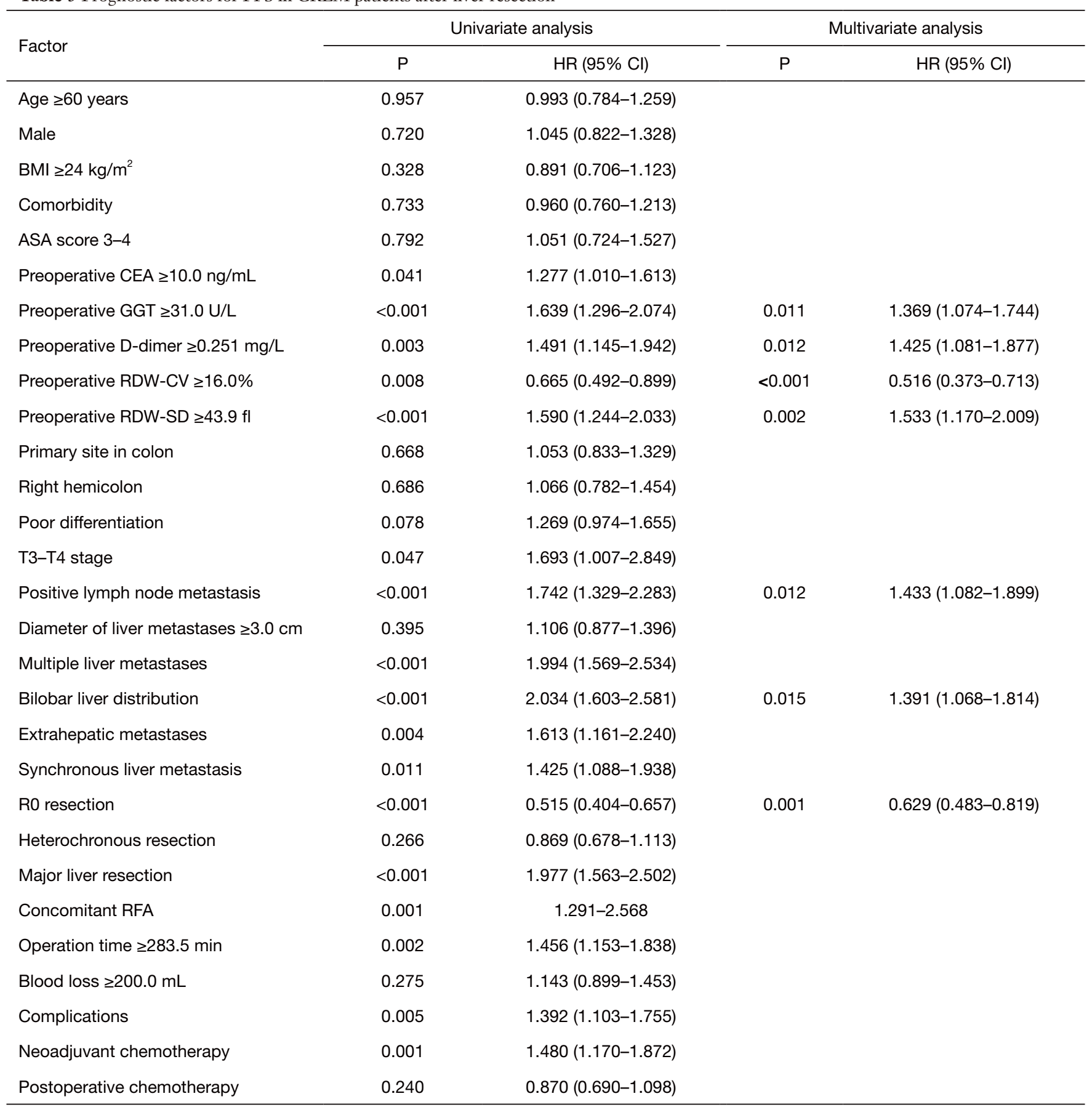

PFS, progression-free survival; CRLM, colorectal liver metastases; CEA, carcinoembryonic antigen; GGT, gamma-glutamy Itranspeptidase; RDW, red cell volume distribution width; NAC, neoadjuvant chemotherapy; CRS, clinical risk-scoring; RFA, radiofrequency ablation. 
A

Points
Preoperative serum D-dimer
Tumor margin
Preoperative RDW-SD
Preoperative RDW-CV
Preoperative serum GGT
Primary lymph node metastasis
Bilobar liver distribution
Total points
1-years survival probability
3-years survival probability
5-years survival probability

B

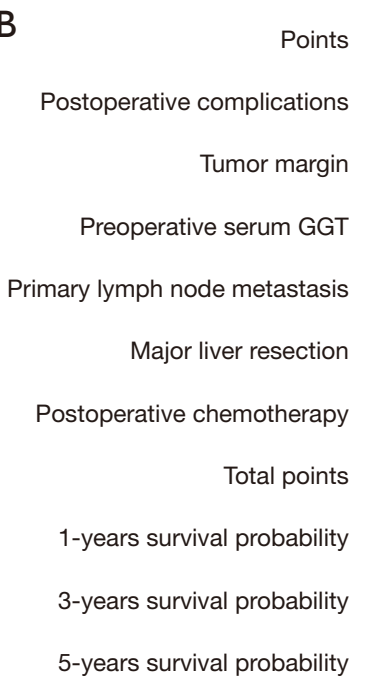

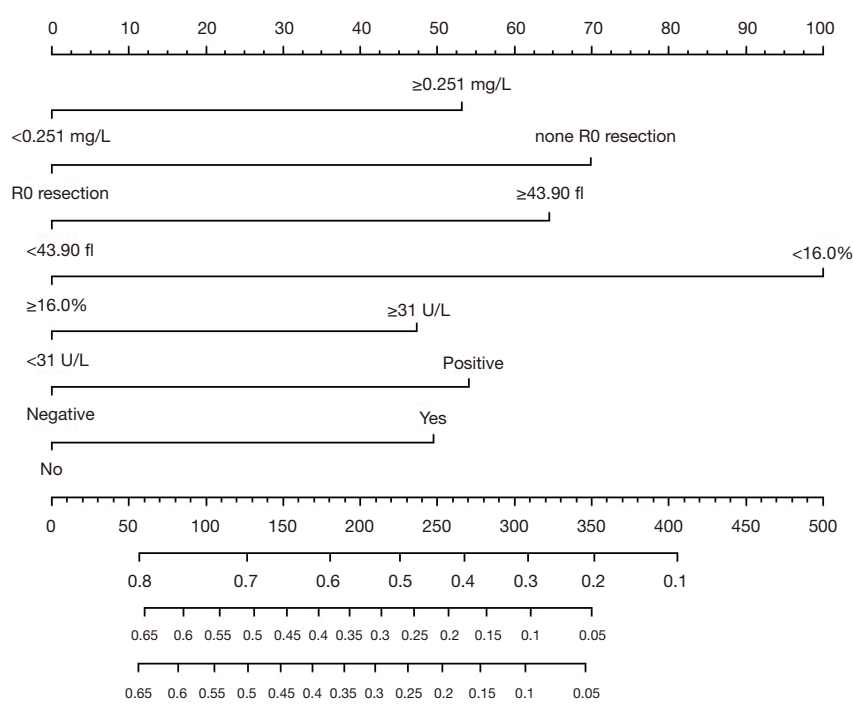

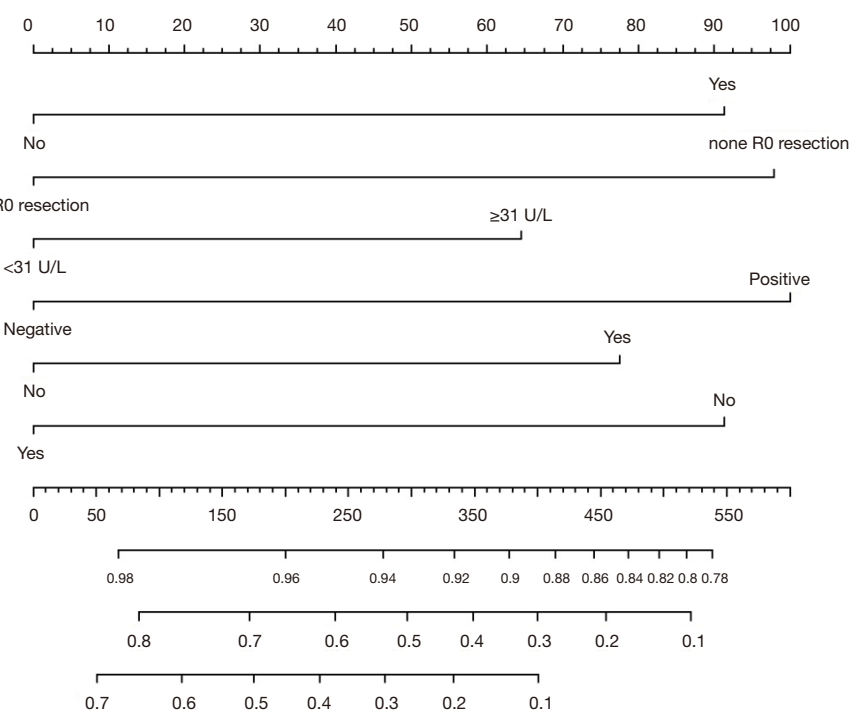

Figure 6 Nomogram for survival. (A) Nomogram for PFS. (B) Nomogram for OS. PFS, progression-free survival; OS, overall survival.

\section{Prognostic factors for $\mathrm{OS}$}

In this study, 163 patients (42.9\%) died. The median OS was 44.1 months (IQR: $37.5-50.7$ ). In the univariable analysis, preoperative GGT $\geq 31.0 \mathrm{U} / \mathrm{L}$, preoperative RDW-CV $<16.0 \%$, preoperative $\mathrm{RDW}-\mathrm{SD} \geq 43.9 \mathrm{fl}$, positive lymph node metastasis, multiple liver metastases, bilobar liver distribution, extrahepatic metastases, non R0 resection, major liver resection, operation time $\geq 283.5$ min, complications, NAC and non-postoperative chemotherapy were related to decreased OS $(\mathrm{P}<0.05)$. Six independent prognostic factors for OS were identified in the multivariable analysis: preoperative GGT $\geq 31.0 \mathrm{U} / \mathrm{L}$ (HR $=1.410,95 \%$ CI: $1.009-1.973, \mathrm{P}=0.044)$, primary lymph node metastasis (HR $=1.703,95 \%$ CI: 1.158-2.503, $\mathrm{P}=0.007)$, R0 resection (HR $=0.594,95 \%$ CI: $0.426-0.827$, $\mathrm{P}=0.002)$, major liver resection $(\mathrm{HR}=1.512,95 \% \mathrm{CI}$ : 1.071-2.133, $\mathrm{P}=0.019)$, complications $(\mathrm{HR}=1.626,95 \% \mathrm{CI}$ : 1.184-2.233, $\mathrm{P}=0.003)$, and postoperative chemotherapy $(\mathrm{HR}=0.615,95 \%$ CI: 0.451-0.839, $\mathrm{P}=0.002)$ (Table 4). 

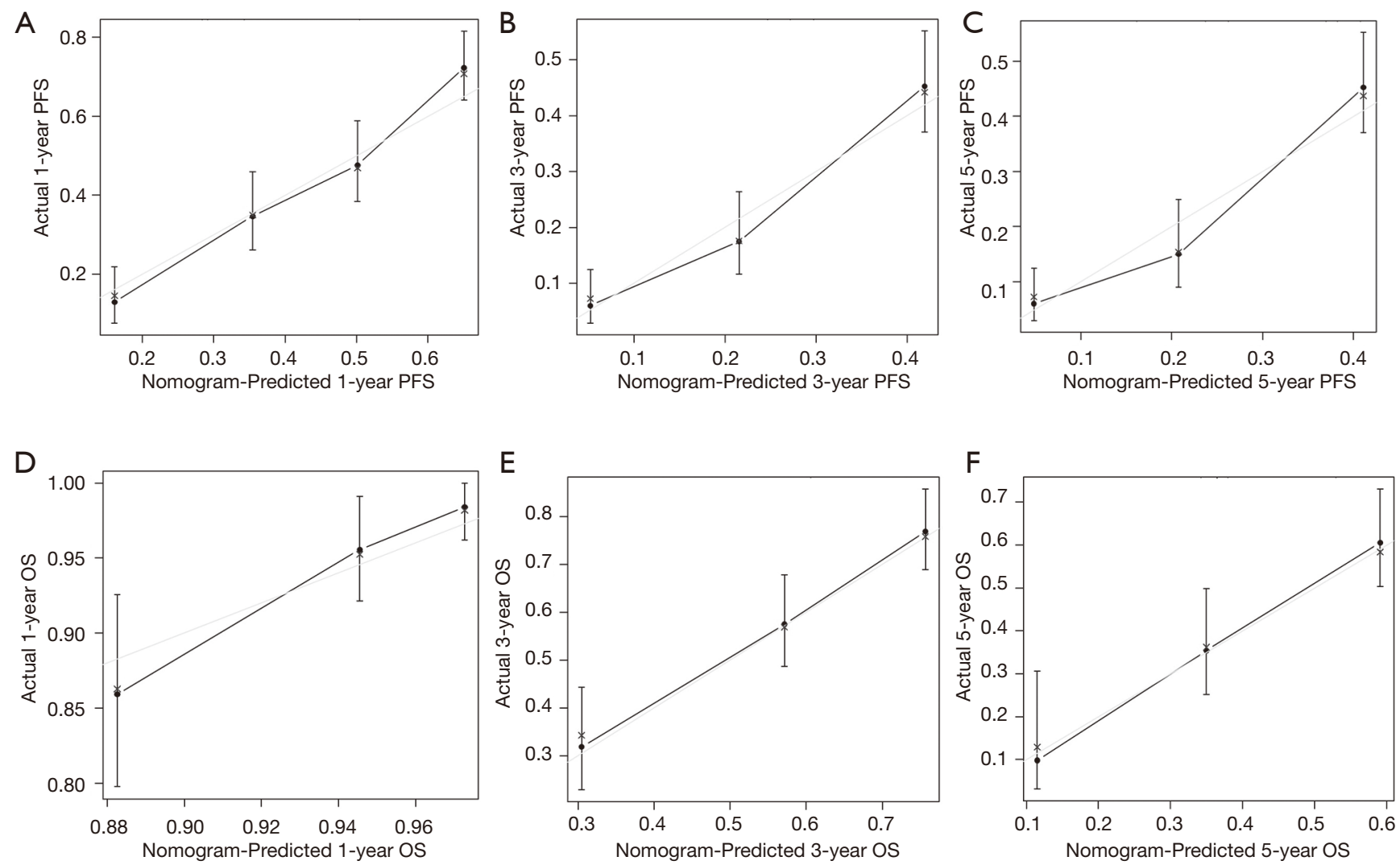

Figure 7 Calibration curves for predicting (A) 1-year, (B) 3-year and (C) 5-year PFS and (D) 1-year, (E) 3-year and (F) 5-year OS. Predicted survival produced by nomogram is plotted on the $\mathrm{x}$-axis, and actual survival is plotted on the $\mathrm{y}$-axis. Dashed lines represent an identical calibration model in which predicted PFS or OS approximate to actual PFS or OS. PFS, progression-free survival; OS, overall survival.

\section{Nomogram for OS Prediction}

A prognostic nomogram for OS after resection with point scales for the six independent prognostic factors described above was constructed (Figure 6). These factors were assigned specific scores as follows: preoperative GGT $\geq 31.0 \mathrm{U} / \mathrm{L}, 53$; primary lymph node metastasis, 54 ; non-R0 resection, 62; major liver resection, 40; complications, 91; and postoperative chemotherapy, 100. The C-statistic for OS prediction was $0.700 \pm 0.021$, which was significantly larger than that of the CRS score $(0.700$ vs. $0.574, \mathrm{P}<0.001)$. A calibration plot for the probability of survival at 1,3 , and 5 years (Figure 7) demonstrated good calibration between the predictions by the nomogram and actual observations. The total risk scores ranged from 0 to 523 for each patient based on the nomogram. According to the total risk scores, the optimal segmentation threshold for dividing patients with CRLM into three subgroups was 335-523 (highrisk group), 242-334 (middle-risk group) and 0-241 (low- risk group). As shown in Figure 5, the high-risk group was associated with significantly worse OS than the middlerisk group ( $\mathrm{P}=0.014$; mOS: 28.1 vs. 35.2 months) and the low-risk group ( $\mathrm{P}<0.001$; mOS: 28.1 months $v s$. not reach). The middle-risk group was associated with significantly worse OS than the low-risk group $(\mathrm{P}<0.001 ;$ mOS: 35.2 months $v s$. not reach).

\section{Discussion}

This study firstly revealed the relationship between preoperative RDW-SD level, preoperative RDW-CV level, and prognosis. The elevated RDW-CV level was associated with postoperative complications and favorable PFS. The elevated RDW-SD level was associated with unfavorable PFS. Additionally, this study established nomograms exclusively based on preoperative blood markers to predict postoperative complications, PFS, and OS in patients with CRLM after liver resection, which could facilitate the 
Table 4 Prognostic factors for OS in CRLM patients after liver resection

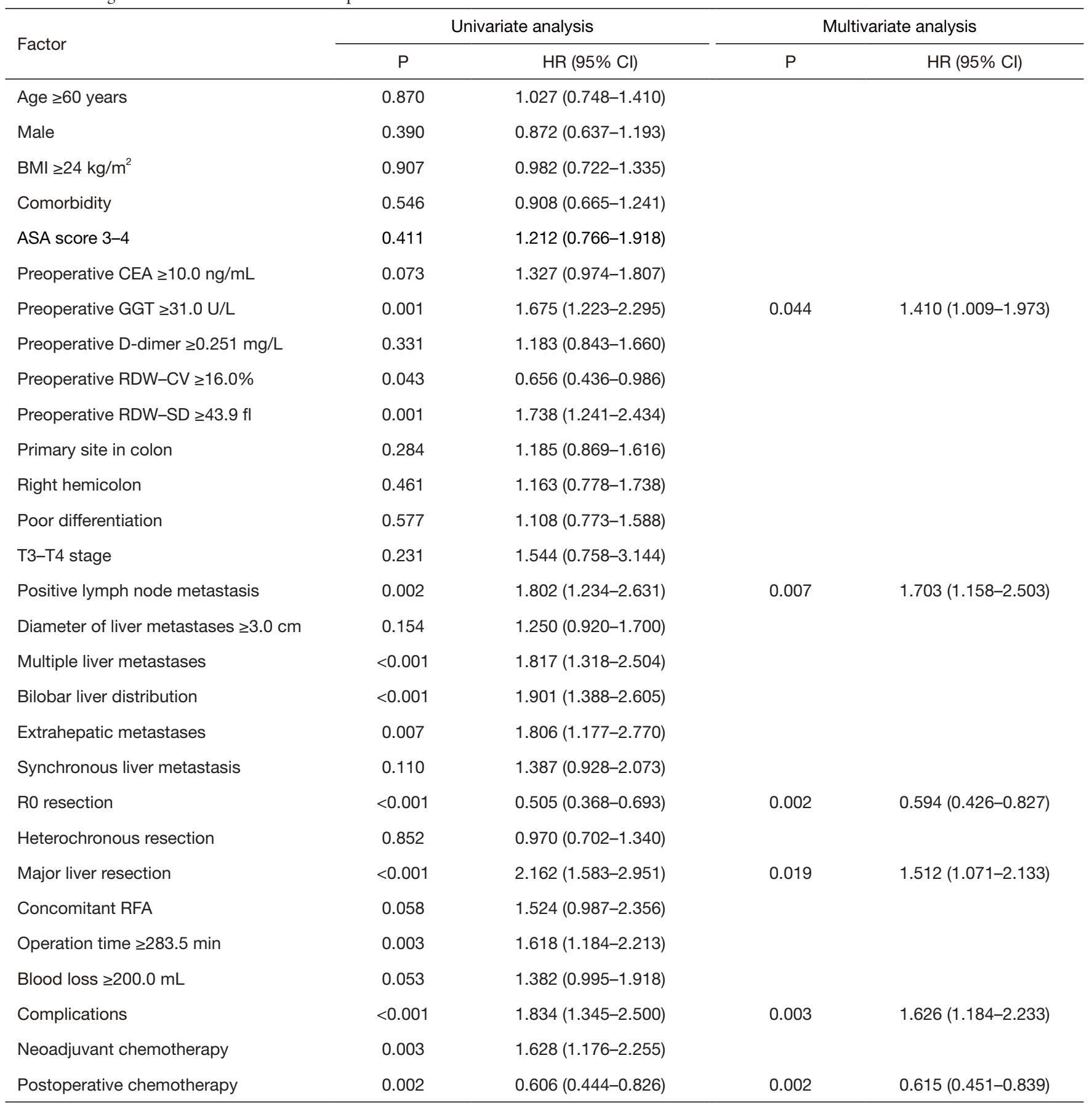

OS, overall survival; CRLM, colorectal liver metastases; CEA, carcinoembryonic antigen; GGT, gamma-glutamy Itranspeptidase; RDW, red cell volume distribution width; NAC, neoadjuvant chemotherapy; CRS, clinical risk-scoring; RFA, radiofrequency ablation. 
individualized evaluation of patients' prognosis, distinguish high-risk patients, and determine optimal clinical management strategies.

RDW level, reflected in RDW-CV and RDW-SD levels, is an indicator of the degree of erythrocyte morphology imbalance in the blood, mirroring the heterogeneity of erythrocyte volume (20). RDW level may change as a result of blood diseases, infectious diseases, malnutrition, and even germline mutations associated with CRC (20-22). Additionally, studies have revealed that elevated RDW level was an independent risk factor for unfavorable survival in patients with CRC and bladder cancer (13-15). Consistent with the previous studies, the results of this study revealed that preoperative elevated RDW-SD level was an unfavorable predictor of PFS and elevated RDW-CV level was related to postoperative complications in patients with CRLM. The mechanisms of the relationship between RDW and outcomes were unclear. There were three possible mechanisms. First, elevated RDW level was correlated with elevated IL-6, circulating cytokines, and tumour necrosis factoralpha which affected aggressive tumour cell behavior (23). Reactive oxygen species and tissue hypoxia increased as RDW level increased, and these factors were associated with high risk of postoperative complications (24). Second, previous studies indicated that RDW level was associated with inflammation markers such as $\mathrm{C}$-reactive protein and erythrocyte sedimentation rate, which demonstrated that RDW level may be an inflammatory marker $(25,26)$. Some studies have shown that inflammatory responses dramatically accelerated tumour growth, progression, and impaired the response to treatment (27). Third, RDW level was associated with dysfunction of various organs. Elevated RDW level indicated that patients may develop organ dysfunction, leading to worse outcome (28-31).

Notably, our study revealed the elevated RDW-CV level was associated with favorable PFS, which was not consistent with the findings of the previous study. Different statistical methods utilized to determine the optimal cutoff values may partially account for the discrepancy in our findings. Firstly, in previous studies, the cutoff values of RDW were determined through ROC analysis or was defined as medians, while in the current study, the cutoff values were obtained through the $\mathrm{X}$ tile analysis which fitting the relationship between prognosis and RDW-CV. Secondly, heterogeneity among different malignancies may drive different predictive value of RDW-CV.

This study also constructed novel nomograms for the prediction of survival. Some nomograms have been developed to predict individual survival probabilities for patients with CRLM undergoing liver resection $(7,8)$. However, the nomograms developed here have the following specific advantages. First, preoperative blood markers were incorporated into the novel nomogram. The main advantage of these blood biomarkers is the ease of their collection from routine blood tests before surgery, so clinicians can tailor management strategies to individual patients conveniently. Second, according to the optimal threshold values, patients with CRLM were stratified into high-risk, middle-risk and low-risk groups. A significant difference in PFS and OS was shown between the risk groups. Clinicians could provide individualized prognostic information and rational suggestions for additional individualized therapy to improve survival according to stratification of patients. Third, CRS score has been widely used to stratify the likelihood of recurrence. However, the CRS score was established based on patients undergoing resection during the 1980s and 1990s (32) and relied solely on traditional clinicopathological factors and was not comprehensive (33). Our nomograms consisting of preoperative blood markers, surgical conditions, and tumour biological characteristics were relatively comprehensive. Furthermore, the nomograms showed desirable performance in discrimination and calibration, outperforming the CRS score. In addition, the high incidence of postoperative complications in patients with CRLM often leads to postponed postoperative recovery, increased medical costs, severe physical stress, and worse quality of life, which highlighted the significance of accurate prediction of postoperative complications. To our knowledge, this was the first nomogram incorporating several preoperative blood markers and surgical condition that could predict the occurrence of postoperative complications in patients with CRLM.

There were several limitations in this study. First, this was a retrospective observational study, which could not confidently infer causality between variables due to potential confounding. The prospective cohort study will help further confirm our conclusions. Second, the variation of surgery was also diverse, and there were some unidentified independent factors that could not be included in the analyses. Third, the nomograms in this study may require further validation from cohorts at other medical centres. Despite these limitations, we believe that our study results provide information applicable to routine clinical practice.

In summary, this study first revealed the relationship between preoperative RDW-SD, preoperative RDW- 
$\mathrm{CV}$, and prognosis. Original predictive nomograms were constructed, especially based on preoperative blood markers, for the survival and postoperative complications of patients with CRLM. The nomograms had relatively good performance and are practical tools to predict prognosis.

\section{Acknowledgments}

Funding: This study was supported by the State Key Project on Infection Diseases of China (Grant No. 2017ZX10201021-007-003), the capital health research and development of special (2018-1-4021), the National Natural Science Foundation of China (81672461 and 81972311), CAMS Innovation Fund for Medical Sciences (CIFMS) (Grant No.2017-12M-4-002), the Non-Profit Central Research Institution Fund of Chinese Academy of Medical Sciences (2019PT310026) and Sanming Project of Medicine in Shenzhen (No. SZSM202011010).

\section{Footnote}

Reporting Checklist: The authors have completed the STROBE reporting checklist. Available at http://dx.doi. org/10.21037/apm-20-2418

Data Sharing Statement: Available at http://dx.doi. org/10.21037/apm-20-2418

Conflicts of Interest: All authors have completed the ICMJE uniform disclosure form (available at http://dx.doi. org/10.21037/apm-20-2418). The authors have no conflicts of interest to declare.

Ethical Statement: The authors are accountable for all aspects of the work in ensuring that questions related to the accuracy or integrity of any part of the work are appropriately investigated and resolved. This study was conducted with approval from the Institute Research Ethics Committee of the Cancer Hospital, Chinese Academy of Medical Sciences (ID:NCC2019C-016). The study conformed to the provisions of the Declaration of Helsinki (as revised in 2013). Informed consents from patients have been obtained.

Open Access Statement: This is an Open Access article distributed in accordance with the Creative Commons Attribution-NonCommercial-NoDerivs 4.0 International License (CC BY-NC-ND 4.0), which permits the non- commercial replication and distribution of the article with the strict proviso that no changes or edits are made and the original work is properly cited (including links to both the formal publication through the relevant DOI and the license). See: https://creativecommons.org/licenses/by-nc-nd/4.0/.

\section{References}

1. Bray F, Ferlay J, Soerjomataram I, et al. Global cancer statistics 2018: GLOBOCAN estimates of incidence and mortality worldwide for 36 cancers in 185 countries. CA Cancer J Clin 2018;68:394-424.

2. Engstrand J, Nilsson H, Strömberg C et al. Colorectal cancer liver metastases - a population-based study on incidence, management and survival. BMC Cancer 2018;18:78.

3. Golubnitschaja O, Polivka J Jr, Yeghiazaryan K, et al. Liquid biopsy and multiparametric analysis in management of liver malignancies: new concepts of the patient stratification and prognostic approach. EPMA J 2018;9:271-85.

4. Kow AWC. Hepatic metastasis from colorectal cancer. J Gastrointest Oncol 2019;10:1274-98.

5. Serayssol C, Maulat C, Breibach F, et al. Predictive factors of histological response of colorectal liver metastases after neoadjuvant chemotherapy. World J Gastrointest Oncol 2019;11:295-309.

6. Chen Q, Wu C, Zhao H, et al. Neo-adjuvant Chemotherapy-Induced Neutropenia Is Associated with Histological Responses and Outcomes after the Resection of Colorectal Liver Metastases. J Gastrointest Surg 2020;24:659-70.

7. Chen Y, Chang W, Ren L, et al. Comprehensive Evaluation of Relapse Risk (CERR) Score for Colorectal Liver Metastases: Development and Validation. Oncologist 2020;25:e1031-41.

8. Liu W, Wang K, Han Y et al. Nomogram predicted disease free survival for colorectal liver metastasis patients with preoperative chemotherapy followed by hepatic resection. Eur J Surg Oncol 2019;45:2070-7.

9. Ma H, Zhang L, Tang B, et al. $\gamma$-Glutamyltranspeptidase is a prognostic marker of survival and recurrence in radiofrequency-ablation treatment of hepatocellular carcinoma. Ann Surg Oncol 2014;21:3084-9.

10. Chen Y, Yu H, Wu C, et al. Prognostic value of plasma $\mathrm{D}$-dimer levels in patients with small-cell lung cancer. Biomed Pharmacother 2016;81:210-7.

11. Liu Z, Guo H, Gao F, et al. Fibrinogen and D-dimer 
levels elevate in advanced hepatocellular carcinoma: High pretreatment fibrinogen levels predict poor outcomes. Hepatol Res 2017;47:1108-17.

12. Bozkaya Y, Yazıcı O. Prognostic significance of gammaglutamyl transferase in patients with metastatic non-small cell lung cancer. Expert Rev Mol Diagn 2019;19:267-72.

13. Ide S, Toiyama Y, Okugawa Y, et al. Clinical significance of an increased red blood cell distribution width in patients with rectal cancer undergoing chemoradiotherapy followed by surgery. Surg Today 2020;50:551-9.

14. Ma W, Mao S, Bao M, et al. Prognostic significance of red cell distribution width in bladder cancer. Transl Androl Urol 2020;9:295-302.

15. Pedrazzani C, Tripepi M, Turri G, et al. Prognostic value of red cell distribution width (RDW) in colorectal cancer. Results from a single-center cohort on 591 patients. Sci Rep 2020;10:1072.

16. Diagnosis, Treatment Guidelines For Colorectal Cancer Working Group C. Chinese Society of Clinical Oncology (CSCO) diagnosis and treatment guidelines for colorectal cancer 2018 (English version). Chin J Cancer Res 2019;31:117-34.

17. Dindo D, Demartines N, Clavien PA. Classification of surgical complications: a new proposal with evaluation in a cohort of 6336 patients and results of a survey. Ann Surg 2004;240:205-13.

18. Tabira Y, Okuma T, Kondo K, et al. Does neoadjuvant chemotherapy for carcinoma in the thoracic esophagus increase postoperative morbidity? Jpn J Thorac Cardiovasc Surg 1999;47:361-7.

19. Camp RL, Dolled-Filhart M, Rimm DL. X-tile: a new bio-informatics tool for biomarker assessment and outcome-based cut-point optimization. Clin Cancer Res 2004;10:7252-9.

20. Salvagno GL, Sanchis-Gomar F, Picanza A, et al. Red blood cell distribution width: A simple parameter with multiple clinical applications. Crit Rev Clin Lab Sci 2015;52:86-105.

21. Di Somma S, Magrini L, Travaglino F, et al. Opinion paper on innovative approach of biomarkers for infectious diseases and sepsis management in the emergency department. Clin Chem Lab Med 2013;51:1167-75.

22. Mahmood NA, Mathew J, Kang B, et al. Broadening of the red blood cell distribution width is associated with increased severity of illness in patients with sepsis. Int J Crit Illn Inj Sci 2014;4:278-82.

23. Hu L, Li M, Ding Y, et al. Prognostic value of RDW in cancers: a systematic review and meta-analysis. Oncotarget
2017;8:16027-35.

24. Xie DX, Rehman SC, Francis DO, et al. Association Between Red Blood Cell Distribution Width and Outcomes of Open Airway Reconstruction Surgery in Adults. JAMA Otolaryngol Head Neck Surg 2019;145:210-5.

25. Lippi G, Targher G, Montagnana M, et al. Relation between red blood cell distribution width and inflammatory biomarkers in a large cohort of unselected outpatients. Arch Pathol Lab Med 2009;133:628-32.

26. Vayá A, Sarnago A, Fuster O, et al. Influence of inflammatory and lipidic parameters on red blood cell distribution width in a healthy population. Clin Hemorheol Microcirc 2015;59:379-85.

27. Marshall HT, Djamgoz MBA. Immuno-Oncology: Emerging Targets and Combination Therapies. Front Oncol 2018;8:315.

28. Felker GM, Allen LA, Pocock SJ, et al. Red cell distribution width as a novel prognostic marker in heart failure: data from the CHARM Program and the Duke Databank. J Am Coll Cardiol 2007;50:40-7.

29. Lippi G, Targher G, Montagnana M, et al. Relationship between red blood cell distribution width and kidney function tests in a large cohort of unselected outpatients. Scand J Clin Lab Invest 2008;68:745-8.

30. Hu Z, Sun Y, Wang Q, et al. Red blood cell distribution width is a potential prognostic index for liver disease. Clin Chem Lab Med 2013;51:1403-8.

31. Wang PF, Song SY, Guo H, et al. Prognostic role of pretreatment red blood cell distribution width in patients with cancer: A meta-analysis of 49 studies. J Cancer 2019;10:4305-17.

32. Fong Y, Fortner J, Sun RL, et al. Clinical score for predicting recurrence after hepatic resection for metastatic colorectal cancer: analysis of 1001 consecutive cases. Ann Surg 1999;230:309-18; discussion 318-21.

33. Margonis GA, Sasaki K, Gholami S, et al. Genetic And Morphological Evaluation (GAME) score for patients with colorectal liver metastases. Br J Surg 2018;105:1210-20.

Cite this article as: Chen Q, Mao R, Zhao J, Bi X, Li Z, Huang Z, Zhang Y, Zhou J, Zhao H, Cai J. Nomograms incorporating preoperative RDW level for the prediction of postoperative complications and survival in colorectal liver metastases after resection. Ann Palliat Med 2021;10(4):4143-4158. doi: 10.21037/apm-20-2418 